\title{
Stress in sputter-deposited Cr films: Influence of Ar pressure
}

\author{
S. Yu. Gracheva) \\ Netherlands Institute of Metals Research, Rotterdamseweg 137, 2628 AL Delft, The Netherlands \\ F. D. Tichelaar and G.C.A.M. Janssen \\ Department of Materials Science and Engineering, Delft University of Technology, Rotterdamseweg 137, \\ 2628 AL Delft, The Netherlands
}

(Received 30 September 2004; accepted 27 January 2005; published online 21 March 2005)

\begin{abstract}
We studied the tensile stress and grain-width evolution in sputter-deposited Cr films with thickness from $20 \mathrm{~nm}$ to $2.7 \mu \mathrm{m}$. Films were deposited in an industrial Hauzer 750 physical vapor deposition machine at $50-80{ }^{\circ} \mathrm{C}$. The films exhibited a columnar microstructure. A power law behavior of the tensile stress as well as of the average grain width with thickness was observed. Both power exponents were strongly dependent on the Ar pressure during deposition. The power exponent $\alpha$ for stress varied from 0.26 to 0.79 for the range of Ar pressures used $\left(5 \times 10^{-3}-2 \times 10^{-2} \mathrm{mbar}\right)$. The mechanism of tensile stress generation is the shrinkage of the grain boundaries. Assuming the same shrinkage of the grain boundaries all through the layer, the stress and the grain width would be inversely proportional. Indeed, the grain width followed the same power law as the stress at low Ar pressure $[\alpha=0.3(1)]$, but not at high Ar pressure $[\alpha=0.58(3)]$. Transmission electron microscopy showed the formation of numerous voids. At higher Ar pressure the void fraction is significantly higher than at low pressure, thereby diminishing stress generation. (C) 2005 American Institute of Physics. [DOI: 10.1063/1.1876579]
\end{abstract}

\section{INTRODUCTION}

Intrinsic stress is an important characteristic of thin films. It has a major effect on the performance of a thin metal coating. The origin of stress in sputter-deposited metal films has been intensively investigated during several decades. It has been established that, basically, the tensile stress in sputter-deposited thin films is due to the attraction between grains at grain boundaries (grain boundary shrinkage) and the compressive stress is generated by ion-peening. ${ }^{1,2} \mathrm{Re}$ cently it was shown for a series of $\mathrm{Cr}$ films deposited at a pressure of $0.3 \mathrm{~Pa}$ that the tensile stress is proportional to the amount of grain boundaries per unit length. ${ }^{3}$ This proportionality requires that grain boundaries undergo the same shrinkage all through the layer. The general validity of this hypothesis can be disputed since it is easy to imagine that shrinkage of the grain boundaries is dependent on, for example, crystallographic orientations of neighboring grains, which may change with thickness.

The microstructure of polycrystalline films deposited by magnetron sputtering is qualitatively described by the structure zone model (SZM) (see Ref. 4 and references therein). SZM is based on experimental observations and differentiates microstructures on the basis of the ratio between the substrate temperature and the melting point of the material, $T_{s} / T_{m}$. As the substrate temperature increases, SZM predicts the microstructure to be, first, rather open columnar (zone I); then competitive growth is predicted, which results in columnar structure with grains widening with thickness (zone T); zone II, at even higher $T_{s}$, predicts much larger columnar grains. However, other parameters, for example, gas pres-

\footnotetext{
a) Author to whom correspondence should be addressed; electronic mail: s.y.grachev@tnw.tudelft.nl
}

sure, level of contamination, and ion bombardment, can influence the growth resulting in the microstructure of another zone. When grown in zone $\mathrm{T}$, grains widen with thickness, while tensile stress decreases. The widening of the grains results from competitive growth of grains with different crystallographic orientations and, consequently, with different growth rates. ${ }^{5}$ Faster growing grains widen on the expense of others. In this way, grains with a certain preferential orientation start to dominate and a certain crystallographic texture is formed.

A number of atomistic growth models as well as stochastic growth equations describe the surface growth in terms of scale invariance. ${ }^{6,7}$ The scaling behavior of grain width and surface roughness with thickness was also observed experimentally: in bcc high-melting point metal films ${ }^{3}$ and in fcc low-melting point metal films produced by sputter deposition $^{8}$ as well as by other methods. ${ }^{9,10}$ The power exponents describing the growth are characteristic parameters of a growing system. However, experimental conditions can influence the overall growth behavior and power exponents as well. The two surface processes influencing the growth of high-melting point metals are surface diffusion and geometric shadowing. While in molecular-beam epitaxy only surface diffusion is relevant, in physical vapor deposition (PVD) both phenomena are of importance. ${ }^{11}$

Cr films are often deposited by PVD in industrial applications. In this paper, we report on the scaling behavior of the tensile stress in sputter-deposited $\mathrm{Cr}$ films and relate it to the grain growth. The experimental conditions corresponded to the border between zone I and zone T. Since no recrystallization occurs during deposition of $\mathrm{Cr}$ at room temperature, the microstructure of a film preserves the growth history. Thus, stress measurements were combined with transmission 


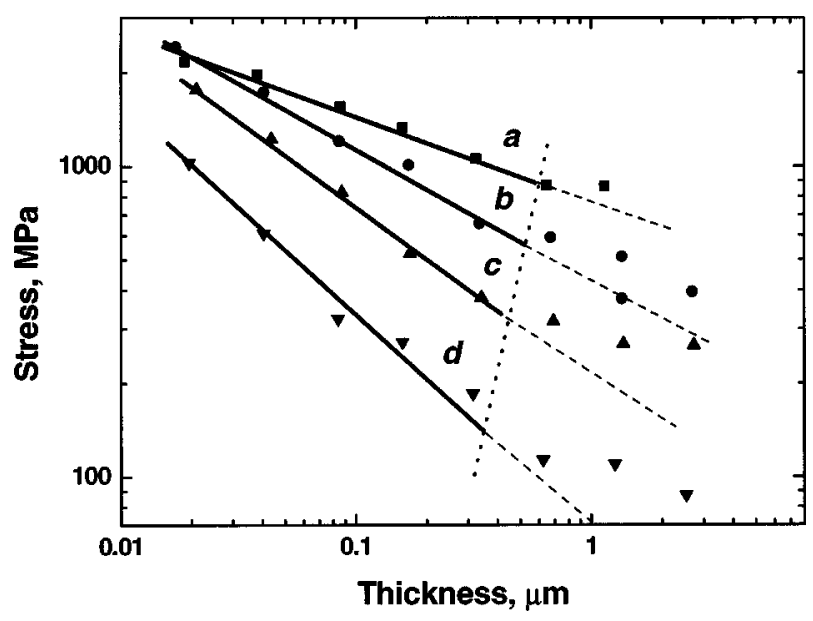

FIG. 1. Stress vs thickness in double-logarithmic scale for samples produced at Ar pressures of (a) $5 \times 10^{-3}$, (b) $7 \times 10^{-3}$, (c) $1 \times 10^{-2}$, and (d) 2 $\times 10^{-2}$ mbar. The slopes of the lines, fitted through the data, are $0.26,0.43$, 0.57 , and 0.79 , respectively.

electron microscopy study of the microstructure. The influence of Ar pressure during sputter deposition on the scaling behavior and on the film microstructure was investigated.

\section{EXPERIMENTAL DETAILS}

Cr films with thickness from $20 \mathrm{~nm}$ to $\sim 2.5 \mu \mathrm{m}$ were deposited in an industrial Hauzer 750 PVD machine. The target plate has dimensions of $600 \times 120 \mathrm{~mm}^{2}$ and is placed at $18-\mathrm{cm}$ distance from the sample holder. Si(100) wafers of $100-\mathrm{mm}$ diameter with a native oxide were used as substrates. The temperature of the substrate during deposition was in the range of $50-80{ }^{\circ} \mathrm{C}$. We applied a dc-power of $5 \mathrm{~kW}$ to the target, which resulted in the deposition rate of $\sim 2 \mathrm{~nm} / \mathrm{s}$, slightly dependent on Ar pressure. Ar pressure was varied in the range between $5 \times 10^{-3}$ and 2 $\times 10^{-2}$ mbar.

Film stress was measured before and immediately after deposition with a wafer curvature method. ${ }^{12}$ The surface morphology was examined with scanning electron microscopy (SEM). X-ray diffraction (XRD) was used to determine crystallographic texture in films. Transmission electron mi-

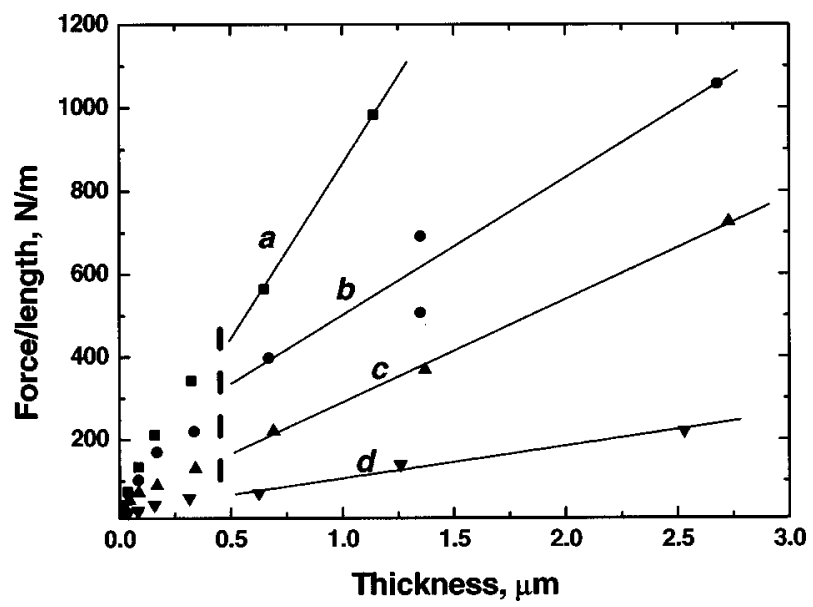

FIG. 2. Force per unit length vs thickness drawn on a linear scale. Data of Fig. 1 were used.

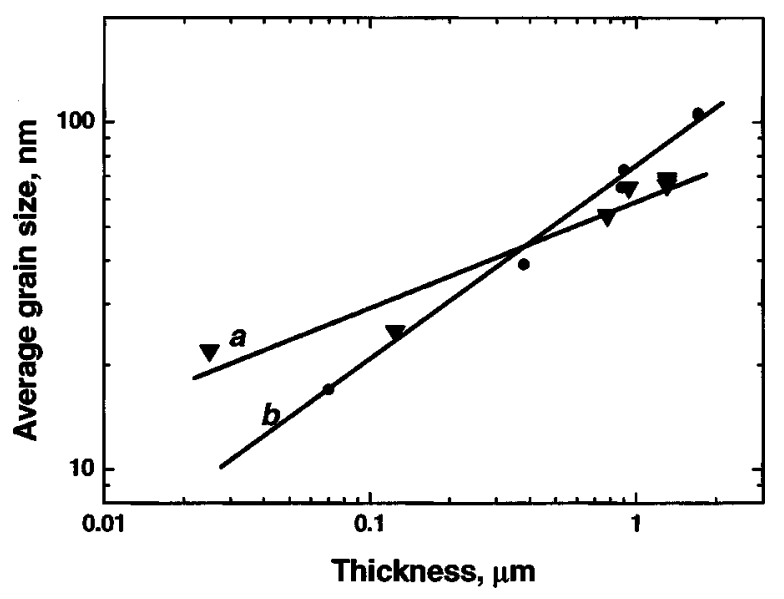

FIG. 3. Average grain width vs thickness on a double-logarithmic scale for two samples grown at the outermost Ar pressures of (a) $5 \times 10^{-3}$ and (b) $2 \times 10^{-2}$ mbar. The slopes are equal to 0.31 and 0.58 , respectively.

croscopy (TEM) was applied to cross sections of two thick samples produced at $5 \times 10^{-3}$ and $2 \times 10^{-2}$ mbar. The brightfield images and selected area diffraction patterns were obtained with a Philips CM30T microscope operating at $300 \mathrm{kV}$. Elemental composition of selected regions of a specimen was done with energy dispersive spectroscopy (EDS).

\section{RESULTS}

Figure 1 shows the development of stress $(\sigma)$ with thickness $(\tau)$ in $\mathrm{Cr}$ films produced at four different Ar pressures: $5 \times 10^{-3}, 7 \times 10^{-3}, 1 \times 10^{-2}$, and $2 \times 10^{-2}$ mbar. The stress curves $\sigma(\tau)$ are presented on a double-logarithmic scale in order to illustrate the power law dependence, $\sigma(\tau) \sim \tau^{-\alpha}$. The power exponent for thinner films varied with Ar pressure in a wide range ( $\alpha=0.26,0.43,0.57$, and 0.79 , respectively). At a thickness of around $1 \mu \mathrm{m}$ the stress curves no longer follow the power law and tend to level off for all Ar pressures. Force per unit length is an integral of stress over thickness of the film and represents the moment exerted by the film on the
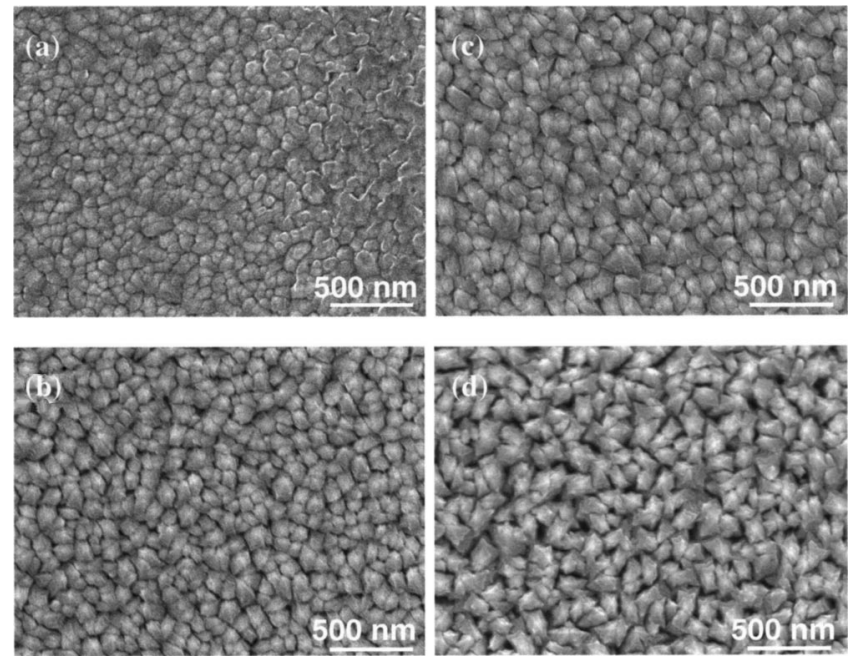

FIG. 4. SEM images of thick samples in series grown at (a) $5 \times 10^{-3}-$, (b) $7 \times 10^{-3}-$, (c) $1 \times 10^{-2}-$, and (d) $2 \times 10^{-2}-$ mbar Ar pressures. For the average grain widths see Table I. 

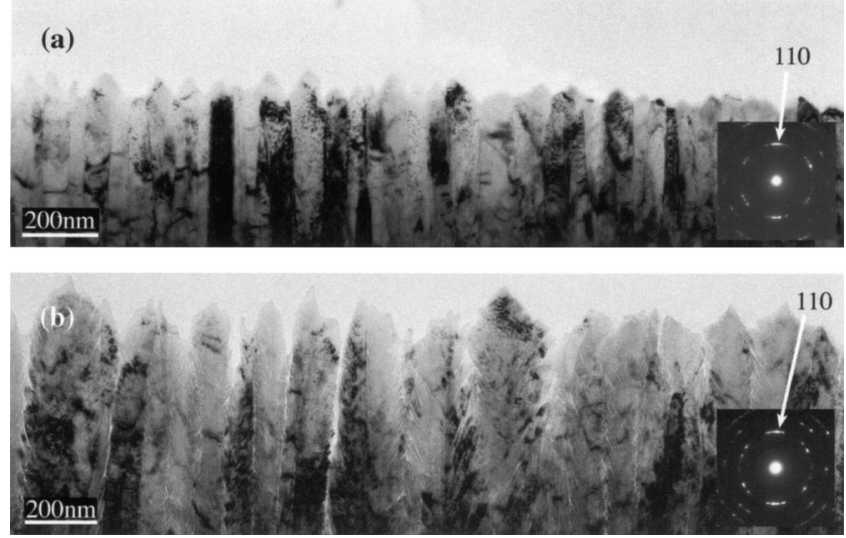

FIG. 5. Brightfield TEM micrographs of specimens produced at (a) low$\left(5 \times 10^{-3} \mathrm{mbar}\right)$ and (b) high-Ar pressure $\left(2 \times 10^{-2} \mathrm{mbar}\right)$. Selected area diffraction patterns are inserted.

substrate. The transition is seen even better when the data are presented as force per unit length versus thickness graph, $\sigma(\tau) \tau$ versus $\tau$, on the linear scale (Fig. 2). The right part of the graph is close to linear, pointing to the absence of a stress gradient, in contrast to the left part.

TEM study showed that the films posses a columnar microstructure (see Fig. 5). The average grain width as a function of distance from the substrate was estimated with TEM for two thick samples. Figure 3 presents the result for samples grown at the outermost Ar pressure. The grain-width increase at a low Ar pressure of $5 \times 10^{-3}$ can be described by a power law with the exponent $(\alpha=0.3 \pm 0.1)$ close to that for the stress evolution in the film $(\alpha=0.31)$. At an Ar pressure of $2 \times 10^{-2} \mathrm{mbar}$, the power exponent for the grain growth is significantly smaller than that for the stress development $(0.58 \pm 0.03$ as compared to 0.79$)$.

It was observed by XRD that the $1.3 \mu \mathrm{m}$-thick samples possessed 110 fiber-type crystallographic texture. The texture was comparable in all cases.

The surface morphology of $1.2-1.3 \mu \mathrm{m}$-thick films was studied with SEM (Fig. 4). The average grain widths are 50, 65,65 , and $100 \mathrm{~nm}$ for samples grown at $5 \times 10^{-3}, 7 \times 10^{-3}$, $1 \times 10^{-2}$, and $2 \times 10^{-2}$ mbar respectively (see also Table I). Another feature observed with SEM is that grains of the sample grown at the highest pressure are faceted and possess sharp edges.

TEM images from the top part of films grown at an Ar pressure of $5 \times 10^{-3} \mathrm{mbar}$ (a) and $2 \times 10^{-2}$ mbar (b) are shown in Fig. 5. The structure of sample $a$ is rather dense in comparison with the porous structure of sample $b .110$ tex-
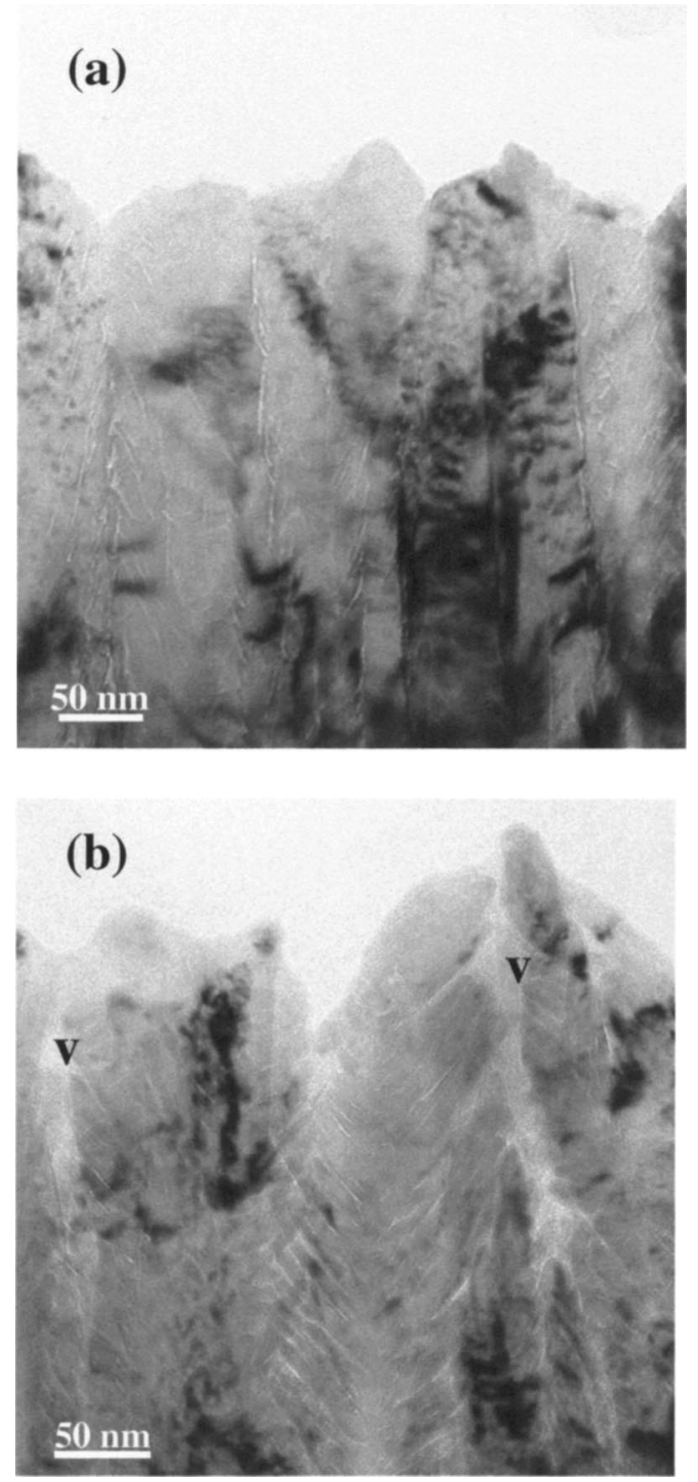

FIG. 6. Brightfield TEM micrographs, taken slightly under focus, from the two samples shown in Fig. 5.

ture was observed with selected area diffraction patterns (insets in Fig. 5), which is common for bcc metals.

Numerous platelike voids, situated both at the grain boundaries and inside the grains, are outlined by Frensel fringes in under-focus conditions (Fig. 6). The grains of sample $b$ consisting of a "stem" and "twigs" are often barely connected. The grains of sample $a$ have a similar, but more dense structure with voids [Fig. 6(a)]. In Fig. 5(a) voids are not readily visible, but in the higher magnification with

TABLE I. Characteristics of thick samples of four series grown at various Ar pressure and power exponents for the series.

\begin{tabular}{lccccc}
\hline \hline $\begin{array}{l}\text { At pressure } \\
(\mathrm{mbar})\end{array}$ & $\begin{array}{c}\text { Thickness } \\
(\mu \mathrm{m})\end{array}$ & $\begin{array}{c}\text { Average grain width } \\
(\mathrm{nm})\end{array}$ & $\begin{array}{c}\text { Stress } \\
(\mathrm{MPa})\end{array}$ & $\begin{array}{c}\text { Exponents for } \\
\text { the stress }\end{array}$ & $\begin{array}{c}\text { Exponents for } \\
\text { the grain width }\end{array}$ \\
\hline $5 \times 10^{-3}$ & 1.14 & 50 & 862 & 0.26 & 0.31 \\
$7 \times 10^{-3}$ & 1.35 & 65 & 375 & 0.43 & $\ldots$ \\
$1 \times 10^{-2}$ & 1.37 & 65 & 269 & 0.57 & $\ldots$ \\
$2 \times 10^{-2}$ & 1.26 & 100 & 110 & 0.79 & 0.58 \\
\hline
\end{tabular}


under-focus [Fig. 6(a)] they are clearly present. Still the grains of sample $a$ are well-attached to each other, which explains a higher tensile stress in the film.

Distinct areas marked in Fig. 6 with "v" are situated at the ends of "twigs," which correspond to wide open areas in between grains that are partly filled with an amorphous material. These areas were investigated with EDS. The analysis showed the presence of a higher percentage of oxygen in this area in comparison with the rest of the film. The enhanced presence of oxygen in the " $v$ " areas suggests that during TEM-specimen preparation oxidation of ion-milling debris ( $\mathrm{Cr}$ ) collected in the voids occurred.

\section{DISCUSSION}

It was proposed in a number of publications that the tensile stress is generated by the shrinkage of the grain boundaries. ${ }^{1,2}$ Assuming the same shrinkage of grain boundaries all over the layer and the power law dependence of the grain width on thickness, the shrinkage itself was estimated in Ref. 3. In this approach, the stress in the layer, $\sigma(\tau)$, should be proportional to the grain-boundary density and inversely proportional to the grain width, $\sigma(t) \sim \mathrm{g}^{-1}(\tau)$. Hence, the decrease of stress and the increase of grain width with thickness should exhibit the same dependence.

Our results confirmed the correlation between the development of stress and grain growth at relatively low Ar pressure. At these conditions, the film microstructure corresponds to zone $\mathbf{T} .^{13}$ However, at a somewhat higher pressure the grain width and the stress evolution exhibit different behavior. At these experimental conditions a large number of voids are formed, which corresponds to zone I. The open microstructure in this case is held responsible for the ineffectiveness of the grain boundaries in stress generation. This explains a lower stress than expected for the given grain width. Thus, the inverse-proportionality between grain width and the tensile stress in the layer was affected by the void formation.

At a low Ar pressure, the kinetic energy of the deposited atoms (mainly atoms, not ions, are deposited) is not reduced by interatomic collisions with the gas phase, and the energy of atoms is distributed in the range of $0.1-10 \mathrm{eV} .^{14}$ This energy promotes surface diffusion, which, in turn, results in the formation of a dense film. In this case, the grain bound- aries are effectively formed and stress is high in such a film. As Ar pressure increases, the flux angular distribution gets wider, and the energy distribution of the deposited atoms shifts to lower values. As voids form due to the lack of diffusion, they are never filled-in since geometric shadowing screens the flux of arriving atoms.

\section{CONCLUSIONS}

The scaling behavior of stress evolution and grain growth in $\mathrm{Cr}$ films was studied. It was observed that the power exponent depends on Ar pressure during deposition. At low Ar pressure, grain width and stress evolve according to the same power law. However, we found that this correlation breaks down when Ar pressure exceeds a certain value. The study of the microstructure showed that the formation of voids in the film is responsible for the ineffectiveness of the grain boundaries in stress generation. Void formation also affects the relationship between the grain width and stress. Furthermore, even for deposition at low pressure the power law breaks down for films thicker than $1 \mu \mathrm{m}$.

\section{ACKNOWLEDGMENTS}

This research was carried out under Project No. MC7.02124 within the framework of the Strategic Research program of the Netherlands Institute for Metals Research.

${ }^{1}$ F. A. Doljack and R. W. Hoffman, Thin Solid Films 12, 71 (1972).

${ }^{2}$ D. W. Hoffman, J. Vac. Sci. Technol. A 12, 953 (1994).

${ }^{3}$ G.C.A.M. Janssen, A. J. Dammers, C. G. M. Sivel, and W. R. Wang, Appl. Phys. Lett. 83, 3287 (2003).

${ }^{4}$ I. Petrov, P. B. Barna, L. Hultman, and J. E. Greene, J. Vac. Sci. Technol. A 21, S117 (2003).

${ }^{5}$ A. van der Drift, Philips Res. Rep. 22, 267 (1967).

${ }^{6}$ A. L. Barabasi and S. H. Stanley, Fractal Concepts in Surface Growth (Cambridge, UK, 1995).

${ }^{7}$ P. Meakin, Phys. Rep. 235, 191 (1993).

${ }^{8}$ A. E. Lita and J. E. Sanchez Jr., Phys. Rev. B 61, 7692 (2000).

${ }^{9}$ N. M. Hasan, J. J. Mallett, S. G. dos Santos Filho, A. A. Pasa, and W. Schwarzacher, Phys. Rev. B 67, 081401(R) (2003).

${ }^{10}$ L. Vazquez, J. M. Albella, R. C. Salvarezza, A. J. Arvia, R. A. Levy, and D. Perese, Appl. Phys. Lett. 68, 1285 (1996).

${ }^{11}$ M. Ohring, Materials Science of Thin Films: Deposition and Structure, (Academic, San Diego, 2002).

${ }^{12}$ P. A. Flinn, D. S. Gardner, and W. D. Nix, IEEE Trans. Electron Devices ED-34, 689 (1987).

${ }^{13}$ P. B. Barna and M. Adamik, Thin Solid Films 317, 27 (1998).

${ }^{14}$ S. M. Rossnagel, C. Nichols, S. Hamaguchi, D. Ruzic, and R. Turkot, J. Vac. Sci. Technol. B 14, 1819 (1996). 